\title{
Tissue-Induced Ultrasonic Wavefront Distortion
}

\author{
Thida Lwin ${ }^{\dagger}$ and William D. O'Brien, Jr. \\ Bioacoustics Research Laboratory, Department of Electrical and Computer Engineering, \\ University of Illinois, 405 North Mathews, Urbana, IL 61801 \\ ${ }^{\dagger}$ Current address: Prucka Engineering, Inc., 8050 El Rio, Houston, TX 77025
}

\begin{abstract}
A quantitative description of wavefront distortion due to tissue inhomogeneities is desirable in order to assist in the development of aberration correction procedures for medical ultrasound imaging. A through-transmission in vitro measurement procedure was used to estimate the ultrasonic wavefront distortions of five different tissue types: bovine liver, porcine liver, bovine skin and bovine muscle for two different orientations (sound direction parallel and perpendicular to fiber orientation). Water and Dow Corning 710 were used for distortionless references. Known thickness tissue samples were vacuum packed in a thin plastic bag at the time of acquisition and were measured in a $20^{\circ} \mathrm{C}$ degassed water tank. Each sample was positioned perpendicular to the beam axis, immediately adjacent to a PVDF membrane hydrophone which was positioned in the $3 \mathrm{MHz}$ circular transducer's focal region to receive the ultrasonic signals that propagated through the tissue sample. For each tissue sample, a total of 625 individual waveforms (25 X 25 rectangular grid in $1 \mathrm{~mm}$ steps) were collected. Three classes of calculations were performed to evaluate tissue inhomogeneities: correlation coefficient between two waveforms, arrival time shift between two waveforms, and the insertion loss for each waveform relative to water. Each was evaluated as a function of two waveform spatial location configurations. One was typical of phased array imaging (linear distance along each data row) and the other was based in eight waveform separation distances through the sample $(1$ to $5.66 \mathrm{~mm})$. Results show that waveform distortion increased as a function of spatial location separation for the tissue samples and was significantly greater for the tissue samples compared to the reference materials.
\end{abstract}

\section{Introduction}

When ultrasonic waves propagate through tissue and tissue layers, they experience wavefront distortion in both amplitude and phase [1]. Phase aberrations induced by spatial tissue inhomogeneities mainly degrade the image quality of phased array ultrasound scanners and have been recognized as a major problem in medical ultrasound imaging [2]. Several methods have been introduced to correct phase aberrations to obtain better quality images [3].

Many experimental studies have been done to determine the degree of tissue inhomogeneities. Krämmer and Hassler [4] concluded that the spatial time-of-flight fluctuations of ultrasound pulses through the abdominal wall were higher for the samples containing more muscle layers than fat layers and that the arrival time fluctuations for liver specimens were of minor importance. Hinkelman et al. [5] measured pulse arrival time delay differences and energy level fluctuations produced by propagation through the human abdominal wall. Similar studies were done on nine different human breast specimens and the average rms values for the arrival time fluctuations for breast specimens were similar to those for abdominal wall specimens of like thickness while the average rms values of energy level fluctuations for breast specimens were greater than that for similarly thick abdominal wall specimens [6].

Observations from this study demonstrate that quantitative information about the degree of tissue inhomogeneity is feasible.

\section{Materials and Methods}

Three bovine liver specimens, three porcine liver specimens, three bovine skin specimens and four bovine muscle specimens (2 each for fiber orientation parallel and perpendicular to sound direction) were obtained from the campus' Meat Science Laboratory. Tissue samples were carefully cut to desired thicknesses, vacuum packed in a thin plastic bag at the time of acquisition and measured in the bag within 1.5 hours of animal death except for the skin samples which were frozen at $-70^{\circ} \mathrm{C}$ prior to thawing and measuring.

All of the experiments were performed in degassed water at $20^{\circ} \mathrm{C}$. A single cycle sinusoid wave, amplified by the RF power amplifier (gain: $55 \mathrm{~dB}$ ), excited a broadband, $3 \mathrm{MHz}$ 
circular transducer (diameter: $2 \mathrm{~cm}$; focal length: $10 \mathrm{~cm} ; 3 \mathrm{~dB}$ depth of focus: $40 \mathrm{~mm}$ ). Tissue sample was placed perpendicular to the beam axis and near the focal region of the transducer. A PVDF membrane hydrophone (active element diameter: $1 \mathrm{~mm}$ ), positioned at the source's focal region on its beam axis and immediately adjacent to the tissue, received the ultrasonic signal that propagated through the tissue. The hydrophone signal was amplified (gain: $20 \mathrm{~dB}$ ), and the output signal was fed into a digital oscilloscope and digitized at $500 \mathrm{MHz}$ (1024 points per waveform). A precision positioning system (accuracy $\approx 2 \mu \mathrm{m}$ ) controlled the movement of the tissue sample. Both the positioning and the data acquisition systems were controlled by the computer via a standard GPIB-IEEE 488 remote-interface.

The measurement procedure was calibrated with Dow Corning 710. Without the tissue sample in water, waveforms through water were acquired. Then, without changing the positions of transducer and hydrophone, 1$\mathrm{cm}, 1.5-\mathrm{cm}$ and $2-\mathrm{cm}$ thick samples of Dow corning 710 were inserted one at a time, as close as possible to the hydrophone, and the waveforms through each sample were acquired. The attenuation coefficients at 2,3 and $4 \mathrm{MHz}$ for Dow Corning 710 were estimated [7]. If the attenuation coefficients agreed to within $\pm 5 \%$ of published values [8], then the calibration procedure was completed and tissue measurements were performed. If not, the hydrophone position was adjusted and the same procedure was repeated.

After the system was calibrated, the positions of the hydrophone and the transducer remained unchanged throughout the experiment. A total of nine waveforms through water only were acquired as reference data. Then, tissue samples were positioned as close as possible to the hydrophone without disturbing the positions of the hydrophone and the transducer, and waveforms through the sample were acquired. The positioning system moved the sample in 1 $\mathrm{mm}$ steps between waveform acquisitions, forming a $25 \times 25$ rectangular grid which covered the specimen's surface area of $2.4 \times 2.4$ $\mathrm{cm}$, producing a total of 625 waveforms.

Calculations were performed on waveform pairs at two different spatial location, and for each waveform pair, correlation coefficient and arrival time shift were estimated as well as insertion loss of each waveform relative to water. The spatial locations of the waveform pairs modeled either linear or $2 \mathrm{D}$ phased array configurations.

For the linear phased array configuration, waveform pairs were confined to a single row of acquired data with the end position of each row common to all waveform pairs of that row.

For the 2D phased array configuration, eight waveform-pair separation distances were evaluated. For each waveform location, the other waveforms were separated a distance of 1 $\mathrm{mm}$ on the rectangular grid. This was repeated for waveform-pair separations of $1.41,2,2.83$, $3,4,4.24$ and $5.66 \mathrm{~mm}$.

The correlation coefficient for waveform pairs was determined from

$$
\rho(\mathrm{x}, \mathrm{y})=\frac{\operatorname{cov}(\mathrm{x}, \mathrm{y})}{\sqrt{\operatorname{var}(\mathrm{x}) \operatorname{var}(\mathrm{y})}}
$$

where $\operatorname{cov}(\mathrm{x}, \mathrm{y})$ is the covariance between the waveform ' $x$ ' and waveform ' $y$ ', $\operatorname{var}(x)$ is the variance of waveform ' $x$ ' and $\operatorname{var}(y)$ is the variance of waveform ' $y$ '.

The time shift between waveform pairs was estimated by using the Matlab command 'xcorr'.

The insertion loss $(I L)$ of waveform pairs, where one of the waveforms was acquired through water, was determined from the magnitude spectrum of the Fourier transform [9] where

$$
I L(\mathrm{~dB})=\mathrm{m}_{\mathrm{W}}-\mathrm{m}_{\mathrm{S}}
$$

where $m_{W}$ and $m_{S}$ are the frequency-dependent magnitude spectrum values for the water waveform and the tissue sample waveform, respectively.

\section{Results}

Results for the linear phased array configuration are tabulated in Table. 1. Results for the $2 \mathrm{D}$ phased array configuration are shown in Figs 1-3.

The mean correlation coefficients and mean rms arrival time differences for water were about the same for the linear and 2D array configurations $(0.995$ and $0.993 ; 1.23 \mathrm{~ns}$ and $1.73 \mathrm{~ns}$ ).

The mean correlation coefficients for tissue samples were between 0.56 and 0.95 for the linear phased array configuration (except for one bovine liver sample and one porcine liver sample), and decreased from 0.98 to about 0.77 as waveform-pair separation distance increased 
from 1 to $5.66 \mathrm{~mm}$ for the 2D phased array configuration. The rms arrival time differences for tissue samples ranged between 9 and $22 \mathrm{~ns}$ for the linear phased array configuration and increased from 3 to $\approx 20-30$ ns as waveform-pair separation distance increased for the $2 \mathrm{D}$ phased array configuration. The rms insertion loss fluctuations for tissue samples at $3 \mathrm{MHz}$ were between 0.3 and $2.5 \mathrm{~dB}$ for the linear phased array configuration and increased from 0.1 to $\approx$ $1.2 \mathrm{~dB}$ as waveform-pair separation distance increased for the 2D phased array configuration.

\section{Discussion}

All of the tissues studied showed appreciable waveform distortion for both phased array configurations. For the 2D phased array configuration, waveform distortion increased as a function of waveform-pair separation distance. The reference media, water, exhibited negligible distortion. Bovine muscle (parallel) had the highest waveform correlation coefficients among the tissue types and porcine liver had the lowest arrival time differences and insertion loss fluctuations (Figs. 1-3). Bovine skin had the lowest waveform correlation coefficients, bovine liver has the highest arrival time differences, and bovine muscle has the highest insertion loss fluctuations (Figs. 1-3).

\section{References}

[1] Q. Zhu and B. D. Steinberg, "Largetransducer Measurements of Wavefront Distortion in the Female Breast," Ultrasonic Imaging, Vol. 14, pp. 276-299, 1992.

[2] G. E. Trahey, P. D. Freiburger, and D. C. Sullivan, "The impact of acoustic velocity variations on target detectability in ultrasonic images of breasts," Investigative Radiology, Vol. 26, pp. 782-791, 1991.

[3] M. O'Donnell and W. E. Engeler, "Correlation-based aberration correction in the presence of inoperable elements," IEEE Trans. Ultrason., Ferroelect., Freq. Contr., Vol. 39, No. 6, pp. 700-707, 1992.

[4] P. Krämmer and D. Hassler, “ Measurement of spatial time-of-flight fluctuations of ultrasound pulses passing through inhomogeneous layers," Proc. of IEEE Ultrason. Symp.,, New York, pp. 939-942, 1987.

[5] L. M. Hinkelman, D. Liu, L. A. Metlay and R. C. Waag, "Measurements of ultrasonic pulse arrival time and energy level variations produced by propagation through abdominal wall," J. Acoust. Soc. $A m$, vol. 95, No. 1, pp. 530-541, 1994.

[6] L. M. Hinkelman, D. Liu, R. C. Waag, Q. Zhu and B. D. Steinberg, "Measurement and correction of ultrasonic pulse distortion produced by the human breast," J. Acoust. Soc. Am., vol. 97, No. 3, pp. 1958-1969, 1995.

[7] F. Dunn and S. A. Goss, Definitions of Terms and Measurements of Acoustical Quantities, J. F. Greenleaf, Ed. Tissue Characterization with Ultrasound, Vol. 1, CRC Press, 1986.

[8] B. Zeqiri, "Reference liquid for ultrasonic attenuation," Ultrasonics, Vol. 27, pp. 314-315, 1989.

[9] M. Insana, J. Zagzebski and E. Madsen, "Improvements in the spectral difference method for measuring ultrasonic attenuation," Ultrasonic Imaging, No. 5, pp. 331-345, 1983.

Table 1. Results for Linear (1D) Phased Array Configuration

\begin{tabular}{|c|c|c|c|c|c|c|c|c|c|c|c|c|c|c|}
\hline & Water & \multicolumn{3}{|c|}{ Bovine Liver } & \multicolumn{3}{|c|}{ Porcine Liver } & \multicolumn{3}{|c|}{ Bovine Skin } & \multicolumn{2}{|c|}{$\begin{array}{l}\text { Bovine Muscle } \\
\text { (parallel) }\end{array}$} & \multicolumn{2}{|c|}{$\begin{array}{l}\text { Bovine Muscle } \\
\text { (perpendicular) }\end{array}$} \\
\hline a & 0.99 & 0.78 & 0.02 & 0.56 & 0.85 & 0.54 & 0.25 & 0.85 & 0.82 & 0.94 & 0.95 & 0.83 & 0.93 & 0.95 \\
\hline$b$ & 1.23 & 15.9 & 21.2 & 15.6 & 19.0 & 22.3 & 16.2 & 11.9 & 11.5 & 9.45 & $10 . \overline{8}$ & 15.9 & 13.9 & 8.81 \\
\hline c & - & $0 . \overline{44}$ & 0.38 & 0.28 & $0 . \overline{33}$ & 0.34 & 0.30 & 1.85 & 2.49 & 2.02 & 1.39 & 1.87 & 0.77 & 1.02 \\
\hline
\end{tabular}

a: mean correlation coefficient

b: RMS arrival time difference, ns

c: RMS insertion loss fluctuation, $\mathrm{dB}$ 


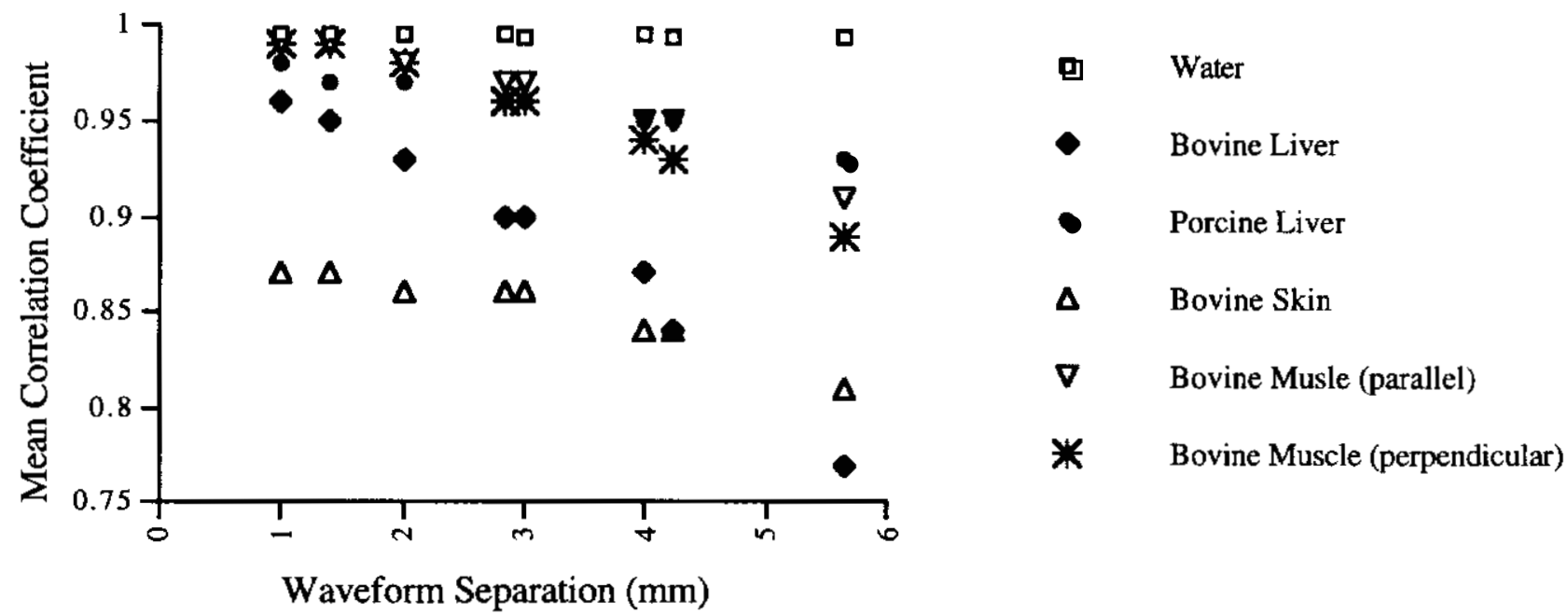

Figure 1. Mean Correlation Coefficients of Water and Tissue Types

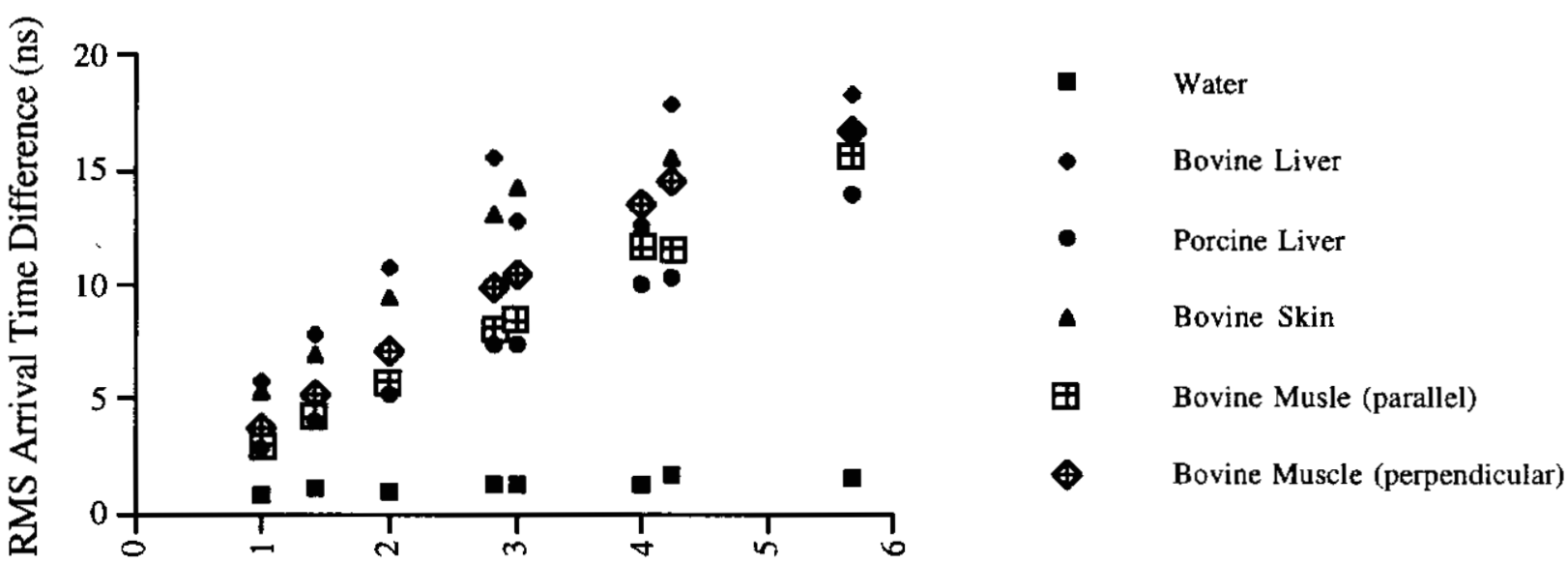

Waveform Separation (mm)

Figure 2. RMS Arrival Time Differences of Water and Tissue Types

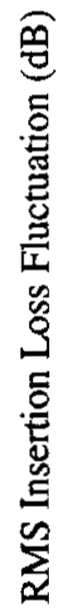

1.25

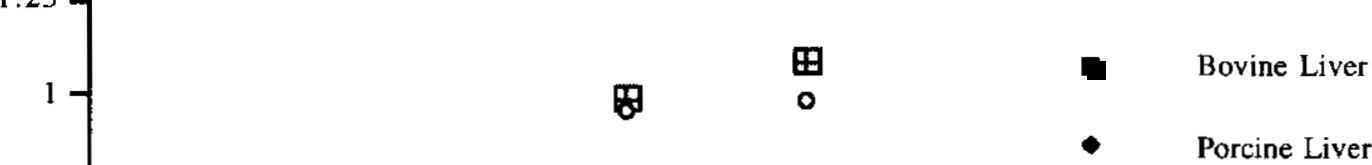

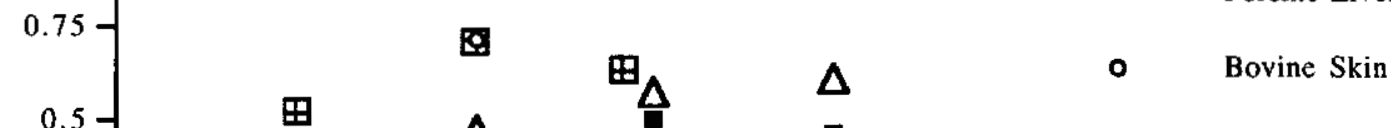

$\begin{array}{llllll} & ⿴ 囗 十 & \Delta & \Delta & \Delta & \Delta\end{array}$

$0.25-\quad \Delta \Delta$ 界

Figure 3. RMS Insertion Loss Fluctuation of Tissue Types 